\title{
Protective Effect of Amantadine in a COVID-19 Patient: Case Report
}

\author{
Albert Cortés Borra* \\ Hospital Vall d'Hebron, Barcelona, Spain
}

The incursion of a new coronavirus isolated for the first time in Wuhan (China) when the first case was diagnosed on November 17, 2019, [1] has been classified by WHO as a global pandemic, [2] in the following months innumerable published and experienced treatment and prophylaxis strategies have been proposed the most recommended being the use of a mask, continuous hand washing, using hydroalcoholic gel and maintaining social distance of 1.5-2 meters. At the pharmacological level, while waiting to have an effective vaccine, a range of drugs from different families have been tested and used including antivirals, antibiotics, anti-malarials, anticoagulants, anti-inflammatories and plasma transfusions, among others [3]. Some of these studies have been surrounded by great controversy, even going so far as to be retracted within a few days of publication of results [4].

In the recent bibliography are few clinical cases related the hypothetic role as preventive use of amantadine, Aranda presents a 57-year-old man with cold symptoms and muscle pain, with elevated blood glucose of $200 \mathrm{mg} / \mathrm{dL}$, was prescribed paracetamol (500 mg every 6 hours) and naproxen (550 mg daily for 5 days) and continued to take $850 \mathrm{mg}$ of metformin twice a day for the treatment of 10-year-old type 2 diabetes. Due to a persistent cough, $500 \mathrm{mg}$ of azithromycin was added for 3 days, but the symptoms continued, and he had to go to his community hospital, where he got a pharyngeal exudate, to do a real-time polymerase chain reaction test for severe acute respiratory syndrome coronavirus 2 (SARS-CoV-2) which was positive, with low $\mathrm{O}_{2}$ saturation that requires oxygen mask. Was treated with $100 \mathrm{mg}$ amantadine twice daily, Asymptomatic family members (wife and daughter 54 and 33 years old, respectively) positive for SARSCoV-2 were prescribed amantadine $100 \mathrm{mg}$ twice daily for 14 days as a preventive measure. The patient's clinical status improved, and oxygen saturation levels gradually improved with combination therapy. By the $6^{\text {th }}$ day, the patient could virtually breathe without the need for oxygen supplementation. The patient was released on the $14^{\text {th }}$ day of treatment. The wife (53-years-old) and daughter (33-years-old) without symptoms but with PCR positive front SARS-CoV-2 were prescribed amantadine $100 \mathrm{mg}$ twice daily for 14 days as a preventive measure, and did not develop symptoms.

Redkak presents 22 patients (10 with multiple sclerosis, 5 with Parkinson's disease and 7 with cognitive impairment) who were tested positive for SARS-CoV-2 and were receiving treatment with either amantadine or memantine on stable registered doses (100 $\mathrm{mg}$ q.d. and $10 \mathrm{mg}$ b.i.d, respectively) for at least 3 months prior to the infection exposure. All patients were tested after reported person-to-person contact with SARS-CoV-2 infected subjects and had viral infection confirmed with PCR test from SARS-CoV-2 in upper and lower respiratory specimens. All of them had two-week quarantine since documented exposure and none developed clinical manifestations of infectious disease. They also did not report any significant changes in neurological status in the course of primary nervous system disease.

This review presents a 75-year-old woman with Parkinson's disease diagnosed 17 years previously is presented. Under medical supervision by her neurologist the patient was receiving opicapone, $50 \mathrm{mg} /$ day (Ongentys $\bullet$ ), pramipexole, $2.1 \mathrm{mg} /$ day (Mirapexin $\bullet$ ), levodopa, 1,000 mg/day, benserazide, $250 \mathrm{mg} /$ day (Madopar $\bullet$ ) and amantadine, $100 \mathrm{mg} /$ day and for hyperthyroidism levothyroxine, $25 \mathrm{mg} /$ day (Euthyrox -). Five years ago, the patient was diagnosed with gastric cancer treated surgically (Billroth II gastrectomy) and with chemotherapy before and after surgery, currently without recurrence of her cancer.

After seven days with a fever oscillating between 37.5$38.8 \mathrm{deg} C$ together with an unusual sporadic cough, mild diarrhea and fatigue, the patient's husband was diagnosed by PCR with COVID-19 which led to his hospital admission with a diagnosis of SARS-CoV-2 infection with bilateral pneumonia. Despite drug treatment and the administration of high-flow oxygen with a Monaghan mask, he died 5 days after admission.

The female patient and wife of the above, who is the object of this clinical case did not present any symptoms of

*Corresponding author: Albert Cortés Borra, RN, MSc, Nursing Supervisor, Valld'Hebron Hospital, Barcelona, Spain, Mail: alcortes@vhebron.net

Accepted: November 11, 2020

Published online: November 13, 2020

Citation: Cortés Borra A (2020) Protective Effect of Amantadine in a COVID-19 Patient: Case Report. Res Rev Infect Dis 3(2):100-101

Copyright: (C) 2020 Cortés Borra A. This is an open-access article distributed under the terms of the Creative Commons Attribution License, which permits unrestricted use, distribution, and reproduction in any medium, provided the original author and source are credited. 
coronavirus infection: Neither fever, nor cough, nor diarrhea, nor anosmia, despite having lived with her husband in a very intimate way, sharing a bed and exposed to the aerosols produced by her husband's persistent cough. Once her husband was admitted to hospital, she was isolated at the home of her daughter, who, after the death of her father, assumed the role of main caregiver. Both her daughter and her daughter's husband tested negative for COVID-19 by PCR.

Based on reports published in the scientific and medical literature and on the personal clinical observations in this personal case, two key questions arise:

1. Is it possible that taking amantadine for months before the contact with SARS-CoV-2 resulted in protection against COVID-19?

2. How is it that despite sharing the same activities with her contacts: Outings in a group of friends - of which 2 also died from COVID-19 - sharing a bed, receiving directly and with no safety distance the aerosols produced by her husband's coughing, she did not become infected or had symptoms?

Amantadine is used to treat the symptoms of Parkinson's disease, multiple sclerosis, and similar conditions. It is also used to control movement problems that are side effects of certain medications used to treat Parkinson's disease. It is also used to prevent symptoms of influenza A virus infection and to treat respiratory infections caused by Influenza A virus [5].

Currently there are a group of investigators who subscribe to the hypothesis that amantadine offers a protective effect to prevent the replication of the virus in patients who are taking it regularly. For example, Tipton \& Wszolek from the Mayo Clinic suggest that "we must explore all possible options to prevent and seek treatment for COVID-19. The use of lowcost and readily available drugs is a low-risk and cost-effective approach. We propose amantadine and memantine as two possible candidates" [6]. Abreu, et al. in the journal "Medical Hypotheses" demonstrate that amantadine blocks the viroporin channel of COVID-19, preventing the release of the viral nucleus in the cell cytoplasm and states that "the use of amantadine when the first symptoms of COVID-19 occur can mitigate the effects of the disease" [7]. In addition, Smieszek, et al. conclude that "amantadine could be used as a potent agent to lower viral load if administered early enough in the course of COVID-19 infection and that the cumulation in lysosomes, if effective, could reduce viral load, decrease organ spread within the host and decrease the severity and progression of associated disease". "They suggest that more studies are needed to examine the role of amantadine" [8]. Rejdak \& Grieb in a study of patients with a range of neurological disorders concluded that "although their study had limitations due to the small sample size and cross-sectional approach, it indicated that the adamantanes amantadine or memantine exert a protective antiviral effect" and that, if confirmed, adamantanes could be useful in limiting SARS-CoV-2 infection and its clinical neurological sequelae" [9]. Cimolai went on to express the view point that, although not commonly discussed, adamantanes should also be reassessed at least in preliminary in vitro studies for various human coronaviruses" [10].

In conclusion, it is the opinion of this author that it is essential to continue this line of investigation, to identify more similar cases, in order to obtain an answers to the two questions alluded to above with a view to providing the Healthcare Systems with the evidence base for the potential use of adamantanes for the prevention of SARS-CoV-2 infection.

\section{References}

1. Chen T, Wu D, Chen H, et al. (2020) Clinical characteristics of 113 deceased patients with coronavirus disease 2019: Retrospective study. BMJ 368: m1091.

2. WHO (2020) Coronavirus disease 2019 (COVID-19) situation report-107.

3. Hung IFN, Lung KC, Tso EYK, et al. (2020) Triple combination of interferon beta-1b, lopinavir-ritonavir, and ribavirin in the treatment of patients admitted to hospital with COVID-19: An open-label, randomised, phase 2 trial. Lancet 395: P1695-P1704.

4. Mehra MR, Ruschitzka F, Patel AN (2020) Retraction-Hydroxychloroquine or chloroquine with or without a macrolide for treatment of COVID-19: A multinational registry analysis. The Lancet 395: P1820.

5. (2020) Amantadina en la enfermedad de Parkinson. Cochrane.

6. Tipton PW, Wszolek ZK (2020) What can Parkinson's disease teach us about COVID-19? Neurol Neurochir Pol 54: 204-206.

7. Abreu GEA, Aguilar MEH, Covarrubias DH, et al. (2020) Amantadine as a drug to mitigate the effects of COVID-19. Med Hypotheses 140: 109755.

8. Smieszek SP, Przychodzen BP, Polymeropoulos MH (2020) Amantadine disrupts lysosomal gene expression; potential therapy for COVID19. BioRxiv.

9. Rejdak K, Grieb P (2020) Adamantanes might be protective from COVID-19 in patients with neurological diseases: Multiple sclerosis, parkinsonism and cognitive impairment. Mult Scler Relat Disord 42: 102163.

10. Cimolai N (2020) Potentially repurposing adamantanes for COVID-19. J Med Virol 9: 531-532.

DOI: $10.36959 / 719 / 575$

Copyright: (C) 2020 Cortés Borra A. This is an open-access article distributed under the terms of the Creative Commons Attribution License, which permits unrestricted use, distribution, and reproduction in any medium, provided the original author and source are credited. 